\title{
Metaanalysis of the Association of Smoking and PTPN22 R620W Genotype on Autoantibody Status and Radiological Erosions in Rheumatoid Arthritis
}

\author{
Lyndsey H. Taylor, Sarah Twigg, Jane Worthington, Paul Emery, Ann W. Morgan, \\ Anthony G. Wilson, and M. Dawn Teare
}

\begin{abstract}
Objective. To investigate the interrelationships among smoking, protein tyrosine phosphatase non-receptor 22 (PTPN22) R620W (rs2476601) genotype, and anticitrullinated peptide antibody (ACPA) status; and among smoking, PTPN22 R620W genotype, and presence of bone erosions overall and separately by ACPA status in patients with rheumatoid arthritis (RA).

Methods. Six studies totaling 2680 patients with RA were included in a Mantel-Haenszel fixed-effects metaanalysis investigating ACPA status and up to 8 studies totaling 3172 patients with RA were included in a Mantel-Haenszel fixed-effects metaanalysis investigating presence of erosive damage.

Results. Evidence was found for an increase in the odds of ACPA positivity for ever smoking (OR $1.56,95 \%$ CI $\left.1.28-1.90, \mathrm{p}=8.5 \times 10^{-6}\right)$, carriage of at least 1 of the PTPN22 risk alleles (OR 1.50, 95\% CI $\left.1.13-2.00, \mathrm{p}=5.5 \times 10^{-3}\right)$ and both ever smoking and carriage of at least 1 of the PTPN22 risk alleles (OR 2.22, 95\% CI 1.69-2.91, p = 8.3 $\times 10^{-9}$ ). There was no evidence of an association between presence of erosive damage and smoking status or carriage of PTPN22 risk alleles when analyzed overall or separately by ACPA status.

Conclusion. This metaanalysis indicates that both smoking and the PTPN22 risk allele are associated with the risk of ACPA positivity. There was insufficient evidence to establish a relationship in either direction between PTPN22 and smoking with erosive damage, despite evidence that ACPA positivity is associated with erosive damage. (First Release May 1 2013; J Rheumatol 2013;40:1048-53; doi:10.3899/jrheum.120784)
\end{abstract}

Key Indexing Terms:

RHEUMATOID ARTHRITIS

SMOKING

A role for genetics in determining rheumatoid arthritis (RA) severity has recently been highlighted in candidate gene association and linkage studies ${ }^{1}$. Possible estimates of the

From the School of Health and Related Research, University of Sheffield, Sheffield; National Institute for Health Research (NIHR) - Leeds Musculoskeletal Biomedical Research Unit, Division of Rheumatic and Musculoskeletal Disease, University of Leeds, Leeds; Musculoskeletal Research Group, University of Manchester, Manchester Academic Health Sciences Centre, Manchester; and the Department of Infection and Immunity, The University of Sheffield Medical School, Sheffield, UK. L.H. Taylor's PhD research is funded by the Medical Research Council. L.H. Taylor, MSc, School of Health and Related Research, University of Sheffield; S. Twigg, MBChB, NIHR-Leeds Musculoskeletal Biomedical Research Unit, Division of Rheumatic and Musculoskeletal Disease, University of Leeds; J. Worthington, PhD, Musculoskeletal Research Group, University of Manchester, Manchester Academic Health Sciences Centre; P. Emery, PhD; A.W. Morgan, PhD, NIHR-Leeds

Musculoskeletal Biomedical Research Unit, Division of Rheumatic and Musculoskeletal Disease, University of Leeds; A.G. Wilson, PhD, Department of Infection and Immunity, The University of Sheffield Medical School; M.D. Teare, PhD, School of Health and Related Research, University of Sheffield.

Address correspondence to L.H. Taylor, School of Health and Related Research, University of Sheffield, Regent Court, 30 Regent Street, SheffieldS1 4DA, UK.E-mail: Lyn.Taylor@sheffield.ac.uk

Accepted for publication March 8, 2013.

\section{HUMAN PTPN22 PROTEIN METAANALYSIS}

heritability of radiological damage, derived using kinship coefficients and identical by descent, were found to be $45 \%$ and $58 \%$, respectively ${ }^{2}$. Large candidate gene studies have reported association of variants of interleukin 6 (IL- 6$)$, $I L-10$, and tumor necrosis factor $\alpha$-induced protein 3 (TNFAIP3) with more severe radiological damage ${ }^{3,4,5}$, as have studies of the RA susceptibility variants in $C D 40^{5}$ and at the TNF-associated factor 1/C5 (TRAF1/C5) locus ${ }^{6}$. Smoking has been linked to production of anticitrullinated protein antibodies (ACPA) in HLA-DRB1 shared-epitope-positive patients. It has also been associated with a worse severity of $\mathrm{RA}^{7,8,9}$. In addition, a single amino acid change R620W (rs2476601) in protein tyrosine phosphatase nonreceptor 22 (PTPN22) has been found to interact with smoking and ACPA positivity ${ }^{10}$. It was therefore hypothesized that carriage of the PTPN22 risk allele may interact with smoking and be associated with more severe RA.

Despite this, 2 recent studies ${ }^{11,12}(\mathrm{n}=532$ and $\mathrm{n}=156$, respectively) found no evidence that smoking increased the risk of being ACPA-positive. One of those studies also reported a protective effect of smoking on the development of bone erosions in subjects who had ever smoked and a 
larger protective effect when subjects ever smoked in combination with carrying at least 1 of the PTPN22 single amino acid changes R620W (rs2476601) ${ }^{11}$.

This protective effect of smoking and the PTPN22 risk allele on radiological damage had not been previously reported. Therefore we conducted a metaanalysis of 6 studies totaling 2680 patients with RA (cases only were included) to investigate the effect of smoking and carrying the PTPN22 risk allele on ACPA positivity status, and a further metaanalysis of 8 studies totaling 3172 patients with RA to investigate the effect of smoking and carrying the PTPN22 risk allele on the presence of erosive damage. In addition, to explore the potential causal pathway between PTPN22, smoking, ACPA positivity, and erosions, we conducted a metaanalysis of 5 studies totaling 2074 patients with RA to investigate the increase in risk of erosions for ACPA-positive versus ACPA-negative patients and provided a subgroup analysis of smoking, PTPN22, and presence of erosive damage for ACPA-positive and ACPA-negative patients separately.

\section{MATERIALS AND METHODS}

RA populations. Subjects meeting the American College of Rheumatology 1987 criteria for RA $^{13}$ with available genotyping data on rs2476601, smoking, and ACPA or erosions were compiled from the following RA populations: Biologics in RA Genetics and Genomics Study Syndicate $(\text { BRAGGSS })^{14}$, Early RA study (ERAS) ${ }^{15}$, Evaluation et Suivi des POlyarthrites Indifférenciées Récentes (ESPOIR) $)^{11}$, Norfolk Arthritis Register (NOAR) ${ }^{16}$, UK RA Genetics (UKRAG; London, Manchester, and Sheffield $)^{17}$, and Yorkshire Early RA (YEAR) ${ }^{18}$. ACPA status was not available for 2 populations (UKRAG London and ERAS), resulting in just 6 populations available for use in the ACPA analysis. All patients were British (with the exception of ESPOIR), white, and age 18 years or older. The Sheffield and BRAGGSS data had average disease duration of about 14 years, whereas all other studies were in early RA (maximum disease duration 2 years). Smoking was dichotomized as "ever smoked" or "never smoked" because of the data available. PTPN22 was classified as "any" (carriage of at least 1 risk allele) versus "no" (carriage of no risk alleles). Studies used their own criteria in the definition of ACPA-positive and negative. For example, UKRAG and Sheffield used $>5.5 \mathrm{U} / \mathrm{ml}$, YEAR used $\geq 5.5 \mathrm{U} / \mathrm{ml}$, or $>10 \mathrm{U} / \mathrm{ml}$ depending on the assay used ${ }^{18}$, and ESPOIR reported to have used $>50 \mathrm{U} / \mathrm{ml}^{11}$. See individual studies for further details. Radiographic examination was performed at recruitment and used to define presence or absence of erosive damage.

Statistical analysis. Patients were categorized into 4 groups by smoking status (ever or never) and presence of the PTPN22 risk allele (any or no). Mantel-Haenszel fixed-effects metaanalyses were performed, comparing each of the 3 categories [ever smoking and no PTPN22 risk allele (EN), never smoking and any PTPN22 risk allele (NA), and ever smoking and any $P T P N 22$ risk allele (EA)] against the reference category of never smoking and carrying no PTPN22 risk allele. The effect of smoking and carrying the PTPN22 risk allele was assessed separately for their effect on the risk of being ACPA-positive and their influence on the risk of any erosive damage. No evidence of study heterogeneity was found based on histograms of the residuals, normal Q-Q plots, $\mathrm{I}^{2}$ statistic, and the chi-square-based Cochran's Q statistic. DerSimonian and Laird random effects metaanalysis models were also produced but are not presented because similar results were obtained.

The increase in risk of erosions for ACPA-positive patients compared to ACPA-negative patients was examined using both the fixed-effect
Mantel-Haenszel method and the random effects DerSimonian and Laird method, because of the strong evidence of heterogeneity between the studies $\left(I^{2}=92.1 \%\right)$.

The effect of smoking and carrying the PTPN22 risk allele on erosive damage was also assessed separately for ACPA-positive and ACPA-negative subjects. Only Mantel-Haenszel fixed-effects metaanalyses were presented because there was no evidence of study heterogeneity and the fixed- and random-effects models obtained similar results.

All metaanalyses were assessed using funnel plots and showed no evidence of publication or selection bias.

There were no adjustments for any covariates because the data were not consistently available across the studies. All analyses were performed using the Metafor Package version 1.6-0 or Meta Package version 1.6-1 (The R Foundation for Statistical Computing, version 2.13.1 $)^{19}$.

\section{RESULTS}

Risk of ACPA positivity. Patients with no PTPN22 risk alleles were 1.56 times as likely to be ACPA-positive if the patient ever smoked compared to never smoked (OR 1.56, $95 \%$ CI $\left.1.28-1.90, \mathrm{p}=8.5 \times 10^{-6}\right)$. Never smokers were 1.5 times as likely to be ACPA-positive if the patient had any $P T P N 22$ risk allele compared to no risk allele (OR 1.50, $95 \%$ CI $\left.1.13-2.00, \mathrm{p}=5.5 \times 10^{-3}\right)$. Patients were 2.22 times as likely to be ACPA-positive if they both ever smoked and had any PTPN22 risk allele compared to never smoking and no risk allele (OR 2.22, 95\% CI 1.69-2.91, $\mathrm{p}=8.3 \times 10^{-9}$ ). The increase in risk is consistent with the product of the single risk factors, suggesting independent effects of ever smoking and having PTPN22 risk alleles. Forest plots of the comparisons by study are shown in Figure 1.

Risk of erosive damage. There was no evidence of risk of erosive damage being associated with whether the patient ever smoked or carried any PTPN22 risk alleles. Forest plots of the comparisons by study are shown in Figure 2 .

We observed an increase in risk of erosive damage for patients who were ACPA-positive compared to ACPA-negative (Figure 3; OR 2.93, 95\% CI 2.33-3.69, $\mathrm{p}=5.6 \times 10^{-20}$ ). Significant evidence of heterogeneity $\left(\mathrm{I}^{2}=92.1 \%\right)$ was present, hence a random effects metaanalysis was also performed but remained statistically significant (OR 2.56, 95\% CI 1.05-6.24, $\mathrm{p}=0.039$ ).

There was no evidence of the risk of erosive damage being associated with whether the patient ever smoked or carried any PTPN22 risk alleles for ACPA-positive subjects (Figure 4) or ACPA-negative subjects (Figure 5).

\section{DISCUSSION}

We assessed the evidence for association between 2 markers of RA disease severity (ACPA positivity and presence of erosive damage) and 2 risk factors (smoking and PTPN22 genotype). The metaanalysis of 6 studies suggests that a history of smoking or carriage of the PTPN22 rs2476601 risk allele is associated with an increased risk of ACPA positivity, particularly when occurring in combination. The second metaanalysis of a total of 8 studies yielded no evidence that a history of smoking or carriage of the 


\section{A}

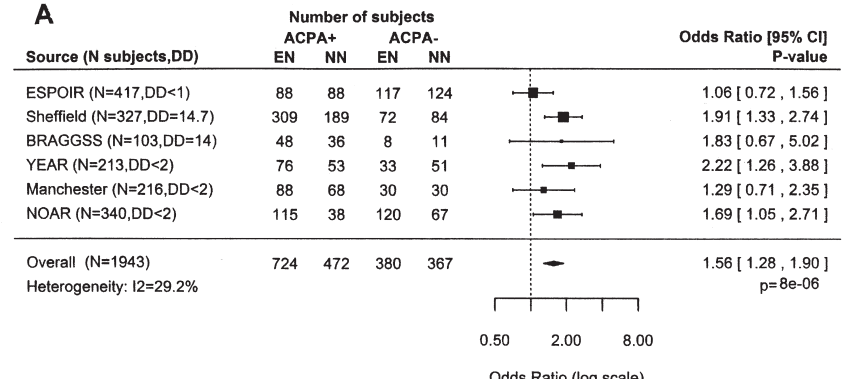

B

\begin{tabular}{|c|c|c|c|c|c|c|c|}
\hline \multirow[b]{2}{*}{ Source (N subjects,DD) } & \multicolumn{4}{|c|}{ Number of subjects } & & & \multirow[b]{2}{*}{$\begin{array}{r}\text { Odds Ratio }[95 \% \mathrm{Cl}] \\
\text { P-value }\end{array}$} \\
\hline & $\begin{array}{l}\text { AC } \\
\text { NA }\end{array}$ & A+ & NA & AN & & & \\
\hline ESPOIR $(N=269, D D<1)$ & 31 & 88 & 26 & 124 & & $\leftrightarrow$ & $1.68[0.93,3.03]$ \\
\hline Sheffield $(N=383, D D=14.7$ ) & 86 & 189 & 24 & 84 & & $\therefore$ & $1.59[0.95,2.68]$ \\
\hline BRAGGSS $(\mathrm{N}=72, \mathrm{DD}=14)$ & 24 & 36 & 1 & 11 & & & $7.33[0.89,60.56]$ \\
\hline YEAR $(N=147, D D<2)$ & 20 & 53 & 23 & 51 & - & - & $0.84[0.41,1.71]$ \\
\hline Manchester $(\mathrm{N}=139, \mathrm{DD}<2)$ & 34 & 68 & 7 & 30 & & 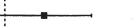 & $2.14[0.85,5.38]$ \\
\hline NOAR $(N=143, D D<2)$ & 15 & 38 & 23 & 67 & - & $\ldots$ & $1.15[0.54,2.47]$ \\
\hline \multirow{3}{*}{$\begin{array}{l}\text { Overall }(N=1153) \\
\text { Heterogeneity: } 12=16.2 \%\end{array}$} & 210 & 472 & 104 & 367 & & - & $1.50[1.13,2.00]$ \\
\hline & & & & & $\Gamma$ & 1 & $p=0.0055$ \\
\hline & & & & & 0.50 & 2.00 & \\
\hline
\end{tabular}

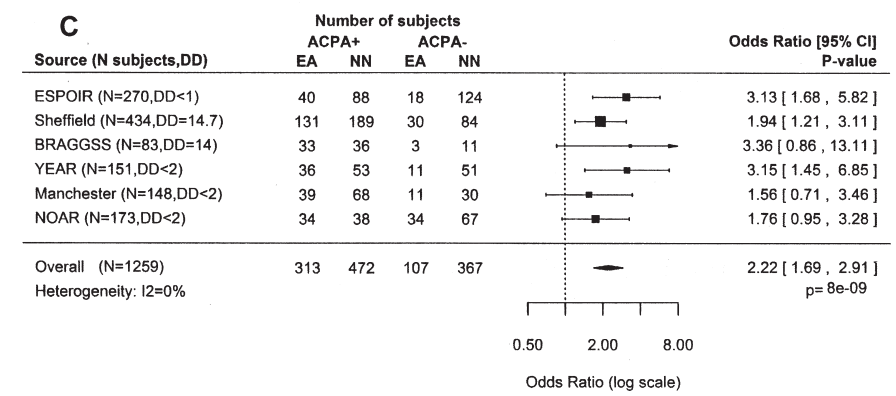

Odds Ratio (log scale)

Odds Ratio (log scale)

Figure 1. Metaanalysis comparing the odds of ACPA-positive versus ACPA-negative for: A. Ever smoking and no PTPN22 risk allele (EN) versus never smoking and no PTPN22 risk allele (NN). B. Never smoking and any PTPN22 risk allele (NA) versus never smoking and no PTPN22 risk allele. C. Ever smoking and any PTPN22 risk allele (EA) versus never smoking and no PTPN22 risk allele. DD is the median or $(<)$ maximum study disease duration in years. Solid lines indicate a 95\% CI and broken line represents an OR of 1. Point estimates are represented by a square whose size is inversely proportional to the standard error of the estimate. ESPOIR: Evaluation et Suivi des POlyarthrites Indifférenciées Récentes; BRAGGSS: Biologics in RA Genetics and Genomics Study Syndicate; YEAR: Yorkshire Early RA study; NOAR: Norfolk Arthritis Register.

A

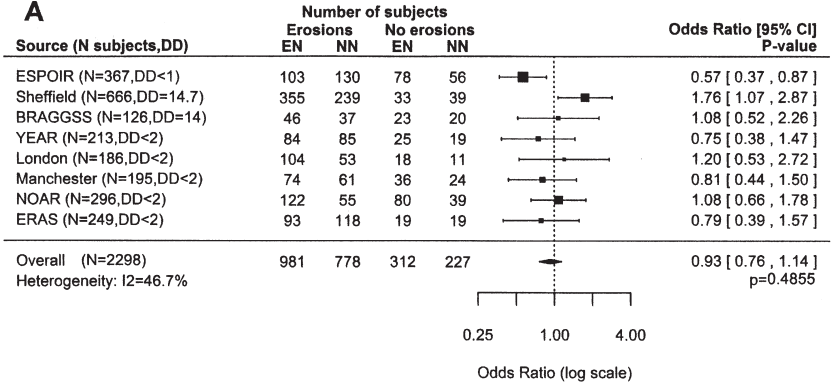

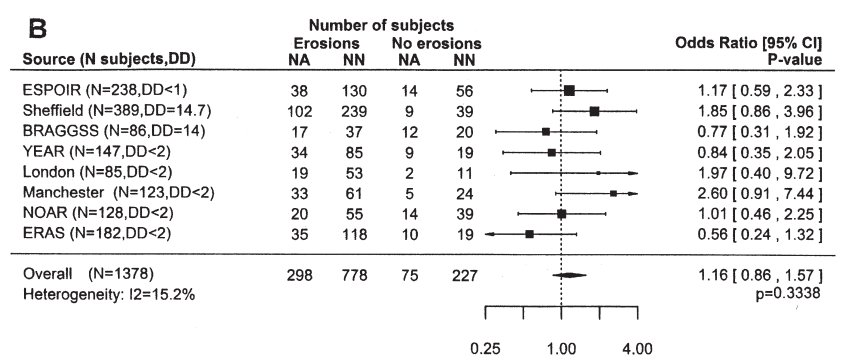

Odds Ratio (log scale)

\begin{tabular}{lcccccc} 
C & \multicolumn{7}{c}{$\begin{array}{c}\text { Number of subjects } \\
\text { Srosions } \\
\text { SA }\end{array}$} & NN & No erosions \\
EA
\end{tabular}

Figure 2. Metaanalysis comparing the odds of erosions versus no erosions for: A. Ever smoking and no PTPN22 risk allele (EN) versus never smoking and no PTPN22 risk allele (NN). B. Never smoking and any PTPN22 risk allele (NA) versus never smoking and no PTPN22 risk allele. C. Ever smoking and any PTPN22 risk allele (EA) versus never smoking and no PTPN22 risk allele. DD is the median or $(<)$ maximum study disease duration in years. Solid lines indicate a 95\% CI and broken line represents an OR of 1. Point estimates are represented by a square whose size is inversely proportional to the standard error of the estimate. The overall summary is represented by a diamond. The width of the diamond corresponds to 95\% CI. ESPOIR: Evaluation et Suivi des POlyarthrites Indifférenciées Récentes; BRAGGSS: Biologics in RA Genetics and Genomics Study Syndicate; YEAR: Yorkshire Early RA study; NOAR: Norfolk Arthritis Register; ERAS: Early RA study. 


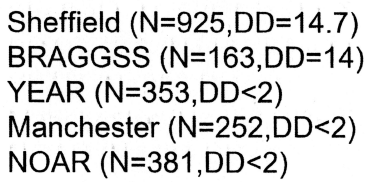

Fixed effect model Random effects model Heterogeneity: I-squared $=92.1 \%$, tau-squared $=0.9412, p<0.0001$

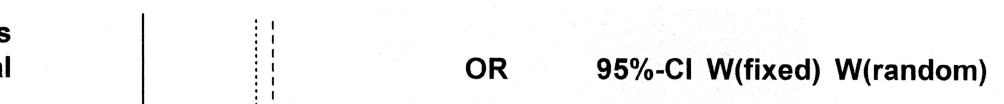

(a)

$\begin{array}{rrrr}670 & 826 & 45 & 99 \\ 89 & 101 & 52 & 6 \\ 167 & 276 & 54 & 77 \\ 148 & 181 & 43 & 71 \\ 143 & 229 & 29 & 15\end{array}$

$1217 \quad 1613 \quad 223 \quad 461$

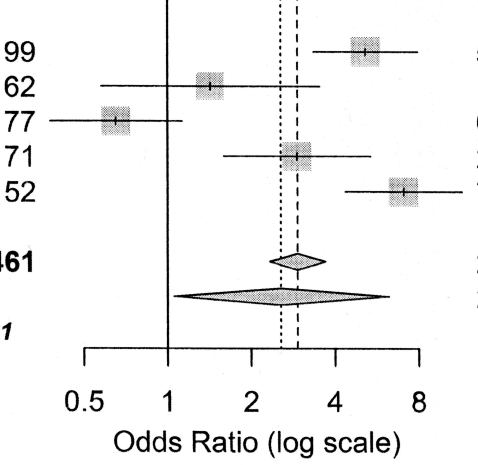

$18.8 \%$

$9.5 \%$

$41.4 \%$

$\begin{array}{lll}2.92 & {[1.59 ; 5.36]} & 14.0 \% \\ 7.05 & {[4.34 ; 11.45]} & 16.3 \%\end{array}$

$\begin{array}{lll}2.92 & {[1.59 ; 5.36]} & 14.0 \% \\ 7.05 & {[4.34 ; 11.45]} & 16.3 \%\end{array}$

$2.93 \quad[2.33 ; 3.69] \quad 100 \%$

$2.56[1.05 ; 6.24]$

$100 \%$
$21.0 \%$

$18.0 \%$

$20.4 \%$

$20.0 \%$

$20.7 \%$

$100 \%$

Figure 3. Metaanalysis comparing the odds of erosions versus no erosions for ACPA-positive versus ACPA-negative subjects. DD is the median or (<) maximum study disease duration in years. Solid horizontal lines indicate a 95\% CI. The size of the box over the point estimate represents the weights determined by the random effect model W(random). Fixed-effects Mantel-Haenszel and random effects DerSimonian and Laird metaanalysis are shown by diamonds whose width corresponds to the 95\% CI. BRAGGSS: Biologics in RA Genetics and Genomics Study Syndicate; YEAR: Yorkshire Early RA study; NOAR: Norfolk Arthritis Register.

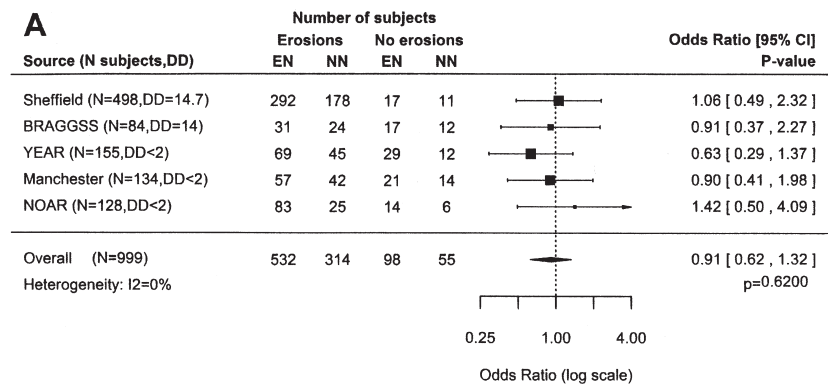

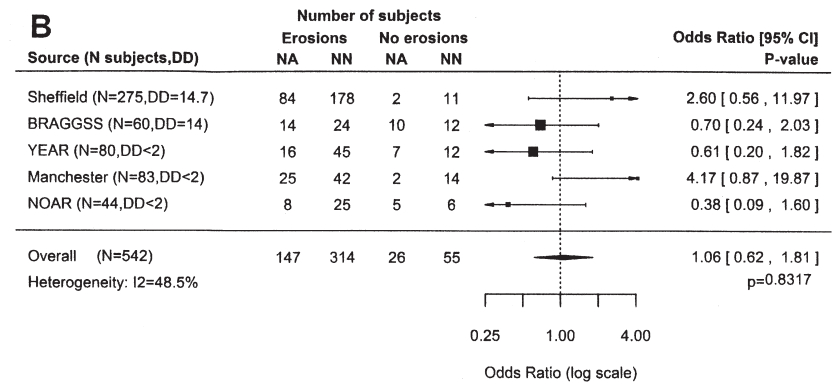

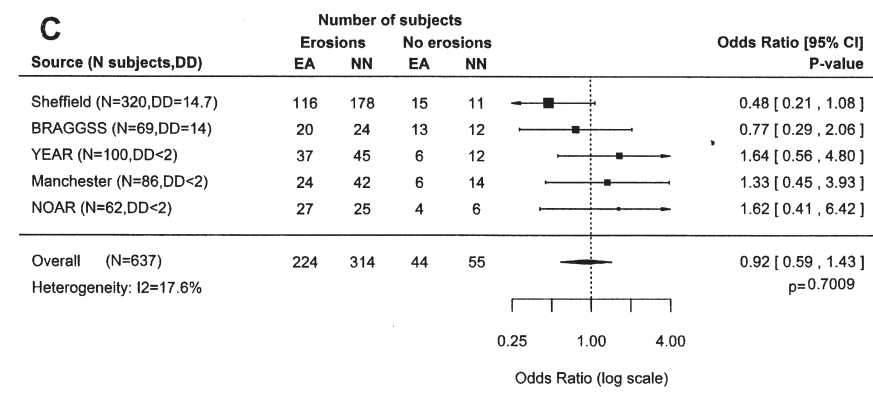

Figure 4. Metaanalysis for ACPA-positive subjects only comparing the odds of erosions versus no erosions for: A. Ever smoking and no PTPN22 risk allele (EN) versus never smoking and no PTPN22 risk allele (NN). B. Never smoking and any PTPN22 risk allele (NA) versus never smoking and no PTPN22 risk allele. C. Ever smoking and any PTPN22 risk allele (EA) versus never smoking and no PTPN22 risk allele. DD is the median or ( $<$ ) maximum study disease duration in years. Solid lines indicate a $95 \%$ CI and broken line represents an OR of 1 . Point estimates are represented by a square whose size is inversely proportional to the standard error of the estimate. The overall summary is represented by a diamond. The width of the diamond corresponds to $95 \%$ CI. BRAGGSS: Biologics in RA Genetics and Genomics Study Syndicate; YEAR: Yorkshire Early RA study; NOAR: Norfolk Arthritis Register.

PTPN22 risk allele, either separately or in combination, affected the risk of erosive damage. We confirmed the association between ACPA positivity and erosions; however, we had insufficient evidence when analyzing separately for ACPA-positive and ACPA-negative subjects that smoking or carriage of the PTPN22 risk alleles was associated with the risk of erosive damage in either of the ACPA subgroups.

A history of smoking alone was found to significantly increase the odds of being ACPA-positive, which is in agreement with previous literature ${ }^{7,8,9}$. While we found supportive evidence that having the PTPN22 risk allele alone and in combination with ever smoking significantly increased the odds of being ACPA-positive, the estimated risk was smaller than previously reported ${ }^{11}$. Given the known link of a more severe disease being associated with ACPA-positive subjects, it is of some reassurance that the 


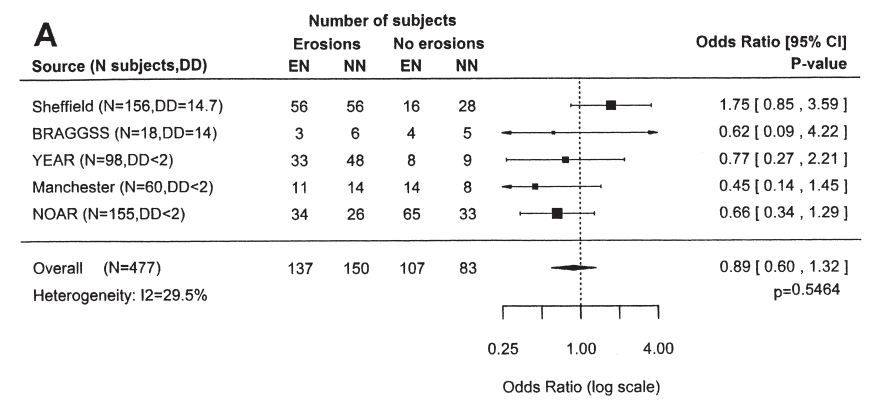

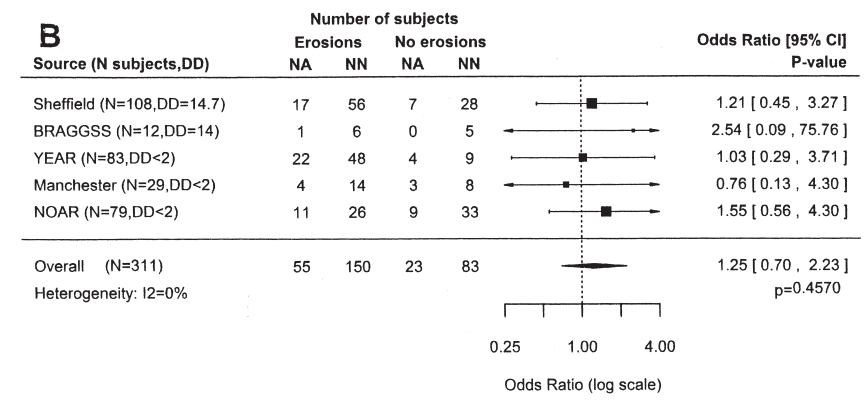

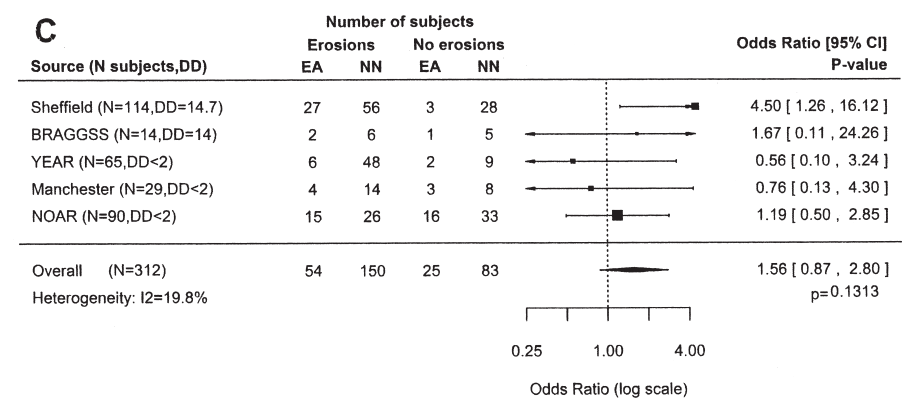

Figure 5. Metaanalysis for ACPA-negative subjects only comparing the odds of erosions versus no erosions for: A. Ever smoking and no PTPN22 risk allele (EN) versus never smoking and no PTPN22 risk allele (NN). B. Never smoking and any PTPN22 risk allele (NA) versus never smoking and no PTPN22 risk allele. C. Ever smoking and any PTPN22 risk allele (EA) versus never smoking and no PTPN22 risk allele. DD is the median or $(<)$ maximum study disease duration in years. Solid lines indicate a 95\% CI and broken line represents an OR of 1 . Point estimates are represented by a square whose size is inversely proportional to the standard error of the estimate. The overall summary is represented by a diamond. The width of the diamond corresponds to $95 \%$ CI. BRAGGSS: Biologics in RA Genetics and Genomics Study Syndicate; YEAR: Yorkshire Early RA study; NOAR: Norfolk Arthritis Register.

recent finding of a protective effect of smoking on erosive status ${ }^{11}$ could not be replicated in this metaanalysis. Although the ESPOIR study used a very different classification of ACPA-positive disease (> $50 \mathrm{IU} / \mathrm{ml}$ ) compared to the other studies, resulting in only $46.4 \%$ of their cases being ACPA-positive, that factor did not appear to result in any great differences in the study conclusions shown in Figures 1B and 1C. However, it could explain why, in Figure 1A, smoking has a smaller increase in the risk of ACPA positivity for the ESPOIR study compared to the other studies.

Of particular interest is the wide variation observed between studies on the estimated effect of smoking on erosive damage (Figure 2A). Possible explanations could be that we were not able to analyze the number of smoking pack-years or fit any potential confounding variables such as the shared epitope or disease duration. Longer durations of smoking and a higher number of cigarettes per day have been associated with a higher risk of ACPA positivity ${ }^{20}$. While analyzing patients as ever or never smoking could have weakened the size of effect in our results, we were still able to detect an association in the ACPA positivity analysis.

Our individual studies are of relatively small sample sizes, particularly when broken down by the binary ACPA, erosive status, smoking, and PTPN22 categories. This results in very small sample sizes when doing the subgroup analysis by ACPA status (Figures 4 and 5) and could explain why no significant effects can be seen in either direction.

Despite 2 of the studies having an average disease duration of 14 years, there was no observable trend in the effect sizes when comparing these 2 studies with the rest of the studies in early RA. Because these studies were not chosen from published literature reporting these comparisons, but were all available data from the UKRAG collaboration, it is unlikely that the metaanalysis is affected by publication or selection bias. A higher risk of RA susceptibility has been reported in patients who are ACPA-positive, carry any PTPN22 risk allele, have at least 1 of the HLA-DRB1 shared epitopes, and are ever smokers ${ }^{21}$. Therefore if data were available, an extension to this work would be advantageous, exploring how the presence of the shared epitope motif affects our estimates of ACPA positivity and erosive damage.

The current data strongly suggest that the risk of being ACPA-positive is increased for patients with RA who have ever smoked and carry any PTPN22 risk allele. We found no evidence to support the recently reported ${ }^{11}$ protective effect of ever smoking and carrying a PTPN22 risk allele on risk of erosive damage. 


\section{ACKNOWLEDGMENT}

We thank John Taylor for his assistance with compiling the data.

\section{REFERENCES}

1. Marinou I, Maxwell JR, Wilson AG. Genetic influences modulating the radiological severity of rheumatoid arthritis. Ann Rheum Dis 2010;69:476-82.

2. Knevel R, Gröndal G, Huizinga TWJ, Visser AW, Jónsson H, Víkingsson A, et al. Genetic predisposition of the severity of joint destruction in rheumatoid arthritis: A population-based study. Ann Rheum Dis 2012;71:707-9.

3. Marinou I, Healy J, Mewar D, Moore DJ, Dickson MC, Binks MH, et al. Association of interleukin-6 and interleukin-10 genotypes with radiographic damage in rheumatoid arthritis is dependent on autoantibody status. Arthritis Rheum 2007;56:2549-56.

4. Scherer HU, van der Linden MPM, Kurreeman FAS, Stoeken-Rijsbergen G, le Cessie S, Huizinga TWJ, et al. Association of the $6 \mathrm{q} 23$ region with the rate of joint destruction in rheumatoid arthritis. Ann Rheum Dis 2010;69:567-70.

5. van der Linden MPM, Feitsma AL, le Cessie S, Kern M, Olsson LM, Raychaudhuri S, et al. Association of a single-nucleotide polymorphism in CD40 with the rate of joint destruction in rheumatoid arthritis. Arthritis Rheum 2009;60:2242-7.

6. Plant D, Bowes J, Potter C, Hyrich KL, Morgan AW, Wilson AG, et al. Genome-wide association study of genetic predictors of anti-tumor necrosis factor treatment efficacy in rheumatoid arthritis identifies associations with polymorphisms at seven loci. Arthritis Rheum 2011;63:645-53.

7. Baka Z, Buzas E, Nagy G. Rheumatoid arthritis and smoking: Putting the pieces together. Arthritis Res Ther 2009;11:238.

8. Lee DM, Phillips R, Hagan EM, Chibnik LB, Costenbader KH, Schur PH. Quantifying anti-cyclic citrullinated peptide titres: clinical utility and association with tobacco exposure in patients with rheumatoid arthritis. Ann Rheum Dis 2009;68:201-8.

9. Pedersen M, Jacobsen S, Klarlund M, Pedersen BV, Wiik A, Wohlfahrt J, et al. Environmental risk factors differ between rheumatoid arthritis with and without auto-antibodies against cyclic citrullinated peptides. Arthritis Res Ther 2006;8:R133.

10. Morgan AW, Thomson W, Martin SG, Carter AM, Erlich HA, Barton A, et al. Reevaluation of the interaction between HLA-DRB1 shared epitope alleles, PTPN22, and smoking in determining susceptibility to autoantibody-positive and autoantibody-negative rheumatoid arthritis in a large UK Caucasian population. Arthritis Rheum 2009;60:2565-76.
11. Salliot C, Dawidowicz K, Lukas C, Guedj M, Paccard C, Benessiano J, et al. PTPN22 R620W genotype-phenotype correlation analysis and gene-environment interaction study in early rheumatoid arthritis: Results from the ESPOIR cohort. Rheumatology 2011;50:1802-8.

12. Ruiz-Esquide V, Gomez-Puerta JA, Canete JD, Graell E, Vazquez I, Ercilla MG, et al. Effects of smoking on disease activity and radiological progression in early rheumatoid arthritis. J Rheumatol 2011;38:2536-9.

13. Arnett FC, Edworthy SM, Bloch DA, McShane DJ, Fries JF, Cooper NS, et al. The American Rheumatism Association 1987 revised criteria for the classification of rheumatoid arthritis. Arthritis Rheum 1988;31:315-24.

14. Maxwell JR, Potter C, Hyrich KL, Barton A, Worthington J, Isaacs $\mathrm{JD}$, et al. Association of the tumour necrosis factor-308 variant with differential response to anti-TNF agents in the treatment of rheumatoid arthritis. Hum Mol Genet 2008;17:3532-8.

15. Young A, Wilkinson P, Talamo J, Dixey J, Jones R, Hunt A, et al. Socioeconomic deprivation and rheumatoid disease: What lessons for the health service? Ann Rheum Dis 2000;59:794-9.

16. Symmons DP, Silman AJ. The Norfolk Arthritis Register (NOAR). Clin Exp Rheumatol 2003;5 Suppl 31:S94-9.

17. Thomson W, Barton A, Ke X, Eyre S, Hinks A, Bowes J, et al. Rheumatoid arthritis association at 6q23. Nat Genet 2007; 39:1431-3.

18. Morgan AW, Robinson JI, Conaghan PG, Martin SG, Hensor EM, Morgan MD, et al. Evaluation of the rheumatoid arthritis susceptibility loci HLA-DRB1, PTPN22, OLIG3/TNFAIP3, STAT4 and TRAF1/C5 in an inception cohort. Arthritis Res Ther 2010;12:R57.

19. Viechtbauer W. Conducting meta-analyses in $\mathrm{R}$ with the Metafor package. J Stat Softw 2010. [Internet. Accessed March 12, 2013.] Available from: www.jstatsoft.org/v36/i03/paper

20. Stolt P, Bengtsson C, Nordmark B, Lindblad S, Lundberg I, Klareskog L, et al. Quantification of the influence of cigarette smoking on rheumatoid arthritis: Results from a population based case-control study, using incident cases. Ann Rheum Dis 2003;62:835-41.

21. Kallberg H, Padyukov L, Plenge RM, Ronnelid J, Gregersen PK, van der Helm-van Mil AH, et al. Gene-gene and gene-environment interactions involving HLA-DRB1, PTPN22, and smoking in two subsets of rheumatoid arthritis. Am J Hum Genet 2007;80:867-75. 